\title{
Duration of SARS-CoV-2 RNA detection in COVID-19 patients in home isolation, Rhineland-Palatinate, Germany, 2020 - an interval-censored survival analysis
}

Sarah Omar ${ }^{1}$, Christoph Bartz ${ }^{2}$, Sabine Becker², Silke Basenach ${ }^{3}$, Sandra Pfeifer ${ }^{3}$, Corinna Trapp ${ }^{4}$, Hildegard Hamm 5 , Hans

Christoph Schlichting ${ }^{6}$, Magdalena Friederichs ${ }^{7}$, Ulrich Koch ${ }^{8}$, Christian Jestrabek ${ }^{9}$, Ernst Hilger ${ }^{10}$, Manfred Vogt ${ }^{11}$, Klaus Jahn ${ }^{12}$, Simiao Chen ${ }^{13,14}$, Till Bärnighausen ${ }^{13,15,16}$, Philipp Zanger ${ }^{11,12,13,17}$, on behalf of the Palatina Public Health Study Group ${ }^{18}$

1. District Public Health Authority, Montabaur, Germany

2. District Public Health Authority, Trier, Germany

3. District Public Health Authority, Neustadt, Germany

4. District Health Authority, Neuwied, Germany

5. District Public Health Authority, Bad Ems, Germany

6. District Public Health Authority, Wittlich, Germany

7. District Public Health Authority, Kirchheimbolanden, Germany

8. District Public Health Authority, Pirmasens, Germany

9. District Public Health Authority, Germersheim, Germany

10. District Public Health Authority, Cochem, Germany

11. Federal State Agency for Consumer and Health Protection Rhineland-Palatinate, Koblenz, Germany

12. Ministry of Health, Federal State of Rhineland-Palatinate, Mainz, Germany

13. Heidelberg Institute of Global Heath, University Hospitals, Heidelberg, Germany

14. Chinese Academy of Medical Sciences and Peking Union Medical College, Beijing, China

15. Harvard Center for Population and Development Studies, Harvard University, Cambridge, United States

16. Department of Global Health and Population, Harvard School of Public Health, Boston, United States

17. Department of Infectious Diseases, Medical Microbiology and Hygiene, University Hospitals, Heidelberg, Germany

18. The participating members of the Palatina Public Health Study Group are acknowledged at the end of the article

Correspondence: Philipp Zanger (philipp.zanger@uni-heidelberg.de)

Citation style for this article:

Omar Sarah, Bartz Christoph, Becker Sabine, Basenach Silke, Pfeifer Sandra, Trapp Corinna, Hamm Hildegard, Schlichting Hans Christoph, Friederichs

Magdalena, Koch Ulrich, Jestrabek Christian, Hilger Ernst, Vogt Manfred, Jahn Klaus, Chen Simiao, Bärnighausen Till , Zanger Philipp, on behalf of the Palatina

Public Health Study Group. Duration of SARS-CoV-2 RNA detection in COVID-19 patients in home isolation, Rhineland-Palatinate, Germany, $2020-$ an interval-

censored survival analysis. Euro Surveill. 2020;25(30):pii=2001292. https://doi.org/10.2807/1560-7917.ES.2020.25.30.2001292

We analysed consecutive RT-qPCR results of 537 symptomatic coronavirus disease (COVID-19) patients in home quarantine. Respectively 2, 3, and 4 weeks after symptom onset, $50 \%, 25 \%$ and $10 \%$ of patients had detectable RNA from severe acute respiratory syndrome coronavirus-2 (SARS-CoV-2). In patients with mild COVID-19, RNA detection is likely to outlast currently known periods of infectiousness by far and fixed time periods seem more appropriate in determining the length of home isolation than laboratorybased approaches.

Most cases of coronavirus disease (COVID-19) are mild [1], making home isolation the standard containment measure for $80 \%$ of patients. Guidance on discontinuation of containment measures is hampered by incomplete knowledge on (i) the level and duration of detecting viral material in different patient groups, (ii) the infectious dose and (iii) the role of RT-qPCR as a proxy measure of infectiousness [2]. This uncertainty explains a significant heterogeneity in the current national recommendations on RNA laboratory testing for severe acute respiratory syndrome coronavirus-2 (SARS-CoV-2) as a tool for discontinuing isolation among countries in the European Union and European Economic Area (EU/EEA) [2].

Here, we present a review of currently published literature on the duration of SARS-CoV-2 RNA detection by $R T-q P C R$ in the respiratory tract in various settings and patients groups. We analyse data of consecutive RT-qPCR results in 537 symptomatic patients with mild COVID-19 in home quarantine, collected from 28 February to 6 June 2020 in Rhineland-Palatinate, Germany. With this research, we aim to close a gap in current knowledge on the duration of RNA detection in patients with mild COVID-19 and inform test-based policy of discontinuing home isolation.

\section{Literature review}

We searched the PubMed online database on 8 April using the terms 'SARS-CoV-2' AND 'shedding' 'NOT faeces' and identified 85 results. Reviewing these papers and their reference lists yielded 11 studies that had analysed sequential RT-qPCR results in populations of 30 or more COVID-19 patients. One of these 11 studies did not provide any summary statistics on temporality [3] and another had been specifically selected for RNA detection of 30 days or longer [4], leaving nine studies 


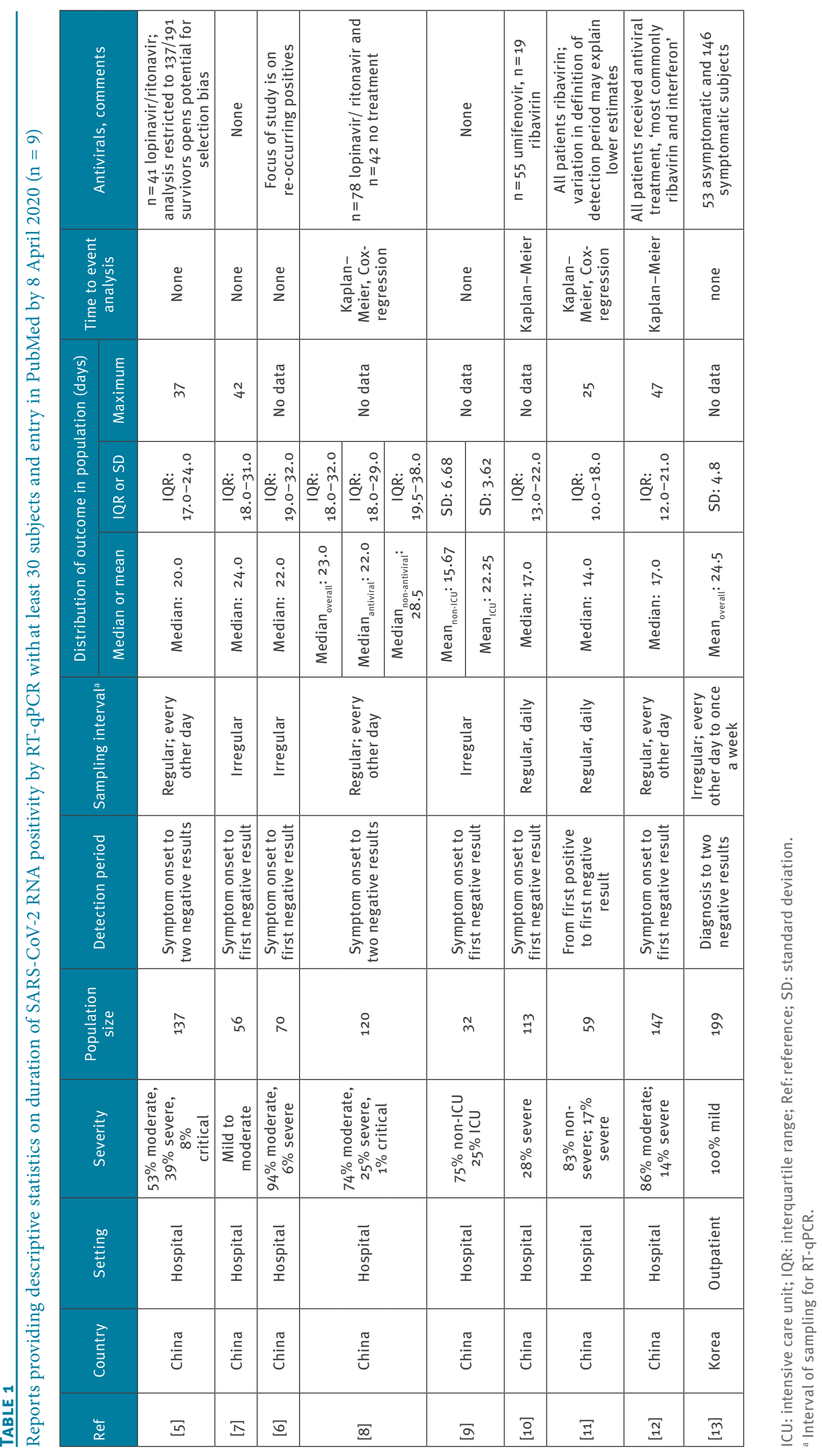


[5-13] in populations of 32-146 COVID-19 patients for detailed comparison of populations, methods and results (Table 1).

All nine studies were conducted in East-Asia (eight in China, one in South Korea) and most of them in a hospital setting $(n=8)$. Substantial proportions of the included patients suffered from severe COVID-19 and received antiviral medication [5-13]. The methodology and reported estimates were heterogeneous. All but two studies $[11,13]$ used the period from symptom onset to time of first negative RT-qPCR result or time to two consecutive negative RT-qPCR results as outcome of interest. Only half of the studies sampled on a regular basis; daily $(n=2)$ or every other day $(n=3)$. Most research, with the exception of one study [13], seemed to use data from irregular (i.e. convenience) sampling. Four studies used survival analysis methods $[8,10-12]$ but none acknowledged the nature of the data by using interval-censoring $[14,15]$. This increases the risk of systematic bias and overestimation, particularly in those studies with irregular sampling intervals $[6,7,9]$. In the seven studies that reported the median duration of RNA detection, the estimates varied from 14 to 24 days. In the remaining two studies, one group reported a mean estimate of 24.5 days. The other study found means of 22.25 and 15.67 days for the intensive care unit (ICU) and non-ICU patient sub-population, respectively. Only one of the nine reviewed studies had looked at COVID-19 patients with mild disease in an outpatient setting; it found viral RNA to be detectable in symptomatic patients for a mean duration of 25.2 days. All nine studies on duration of RNA detection studied small samples, explaining wide measures of spread around the reported estimates. As far as we are aware, there are currently no published data on the duration of RNA positivity in the upper respiratory of patients with mild COVID-19 that could inform a public health assessment of RT-qPCR as a tool for monitoring home isolation.

\section{Retrospective study using routine data from district public health authorities}

Rhineland-Palatinate is one of 16 federal states in Germany and has a population of about 4 million people. Containment of mild COVID-19 cases is managed by 24 district public health authorities (DPHA) responsible for 35 districts. All 24 DPHA received an invitation to retrospectively enter into a EUSurvey database (https://ec.europa.eu/eusurvey/home/welcome) information on demographic characteristics and consecutive RT-qPCR results ('positive', 'negative' or 'equivocal') of COVID-19 patients who were in home isolation. No personal identifying information was stored in the online database.

A person was included in the analysis (i) if they were a laboratory-confirmed, RT-qPCR-positive case of COVID-19, (ii) if they were in home isolation between 28 February 2020 (date of first notified COVID-19 case in Rhineland-Palatinate) and 6 June 2020 (end of study period), (iii) if they had tested negative in RT-qPCR in at least one upper respiratory tract sample provided after diagnosis and before 6 June 2020, and (iv) if they had symptoms of COVID-19 before the first (i.e. diagnostic) RT-qPCR test.

For observations with missing information $(n=98)$, we imputed the date of symptom onset by subtracting the average delay of notification of 5.56, rounded to 6 full days. The latter was calculated from all statutory notifications made by 6 June 2020 that had data on both date of onset and date of statutory notification.

Data were extracted from the online platform on 6 June 2020, imported into Stata, cleaned, and RT-qPCRresults were binarised by recoding 'equivocal' to 'positive'. We used interval-censored survival analysis ('stintreg' command, Stata 15) to estimate survival time, with the left margin of the censoring interval being days between date of onset and last positive swab and the right margin being days between date of onset and first negative swab. Time ratios comparing the median time to RNA negativity in the exposed with that in the unexposed were calculated for several exposure variables. Goodness of fit of different parametric survival models to our data was assessed using the Akaike information criterion [16] and visually by plotting the cumulative hazard function [17] against the Cox-Snell residuals.

Since RT-qPCR analyses were provided by different microbiology laboratories, information on type of RT-qPCR assay, cycle threshold (CT) or raw data on individual CT values were not available. To account for the additional level of variation introduced by the use of different RT-qPCR assays in different microbiology laboratories, we adjusted all regression analyses for clustering by DPHA.

\section{Ethical statement}

All data presented were collected in response to the SARS-CoV-2 pandemic and in accordance with the German Infection Protection Act. Institutional review and individual informed consent was not sought.

\section{Duration of RNA positivity}

Sixteen of 24 district public health authorities in Rhineland-Palatinate submitted data for overall 603 patients. On further review, 53 of them were asymptomatic and 13 had missing information on symptoms, leaving 537 symptomatic COVID-19 patients in home isolation for further analysis (Table 2).

Figure 1 displays key statistics of the number of RT-qPCR tests and time periods analysed for this study. A generalised gamma distribution fit our data best (Supplementary Table S1 and Supplementary Figure). We found that respectively $50 \%, 25 \%$, and $10 \%$ of patients were still positive for SARS-CoV-2 RNA 2, 3 and 4 weeks after the onset of symptoms. At 14 days after onset, the earliest moment to discontinue 


\section{TABLE 2}

Baseline characteristics of symptomatic COVID-19 patients, Rhineland-Palatinate, Germany, 28 February-6 June 2020 $(\mathrm{n}=537)$

\begin{tabular}{|c|c|c|c|}
\hline & $\mathbf{n}$ & \multicolumn{2}{|c|}{$\%$} \\
\hline Male & 233 & \multicolumn{2}{|c|}{$43 \cdot 4$} \\
\hline Female & 304 & \multicolumn{2}{|c|}{56.6} \\
\hline Date of onset unknown ${ }^{a}$ & 98 & \multicolumn{2}{|c|}{18.2} \\
\hline At least one 'equivocal' laboratory result recoded as 'positive' & 17 & \multicolumn{2}{|c|}{3.2} \\
\hline Immunosuppression ${ }^{b}$ & 7 & \multicolumn{2}{|c|}{1.3} \\
\hline \multicolumn{4}{|l|}{ Treatment before home isolation } \\
\hline Inpatient & 67 & \multicolumn{2}{|c|}{12.5} \\
\hline Outpatient & 425 & \multicolumn{2}{|c|}{79.1} \\
\hline Unknown & 45 & \multicolumn{2}{|c|}{8.4} \\
\hline \multicolumn{4}{|l|}{ Epidemiological context at time of diagnosis } \\
\hline Healthcare staff & 81 & \multicolumn{2}{|c|}{15.1} \\
\hline Patient in hospital & 19 & \multicolumn{2}{|c|}{$3 \cdot 5$} \\
\hline Nursing home resident & 18 & \multicolumn{2}{|c|}{$3 \cdot 4$} \\
\hline Community ${ }^{c}$ & 398 & \multicolumn{2}{|c|}{74.1} \\
\hline \multirow[t]{2}{*}{ Unknown } & 21 & \multicolumn{2}{|c|}{3.9} \\
\hline & $\mathbf{n}$ & Median & IQR \\
\hline \multicolumn{4}{|l|}{ Age in years (in five equally sized groups) } \\
\hline $1 \mathrm{st}$ & 114 & 26.0 & $21.0-29.0$ \\
\hline $2 n d$ & 110 & 38.0 & $35.0-41.0$ \\
\hline $3 r d$ & 108 & $49 \cdot 5$ & $46.0-51.5$ \\
\hline $4^{\text {th }}$ & 105 & 57.0 & $55.0-60.0$ \\
\hline $5^{\text {th }}$ & 100 & 71.0 & $64.0-81.0$ \\
\hline \multicolumn{4}{|l|}{ RT-qPCR } \\
\hline Median number of tests per subject until first negative test & 537 & $2.0^{d}$ & $2.0-3.0$ \\
\hline Median time to first negative test in days & 537 & $20.0^{\mathrm{e}}$ & $16.0-28.0$ \\
\hline Length of censoring intervals ${ }^{f}$ in days & 537 & $10.0^{\mathrm{g}}$ & $7.0-15 \cdot 0$ \\
\hline
\end{tabular}

IQR: interquartile range.

amputed by date of notification minus delay of notification of 6 days.

${ }^{b}$ Pregnancy $(n=2)$, renal transplant and dialysis $(n=2)$, cancer $(n=2)$, rheumatoid arthritis $(n=1)$.

' Not belonging to an institutional transmission context or cluster.

dMinimum-maximum: 2-9 tests.

e Minimum-maximum: 1-68 days.

${ }^{\mathrm{f}}$ Defined as time from last positive to first negative test result in days.

g Minimum-maximum: 1-68 days. 
Number of samples analysed per subject and days until first PCR-negative swab in home-isolated COVID-19 patients, Rhineland-Palatinate, Germany, $2020(n=537)$

A.

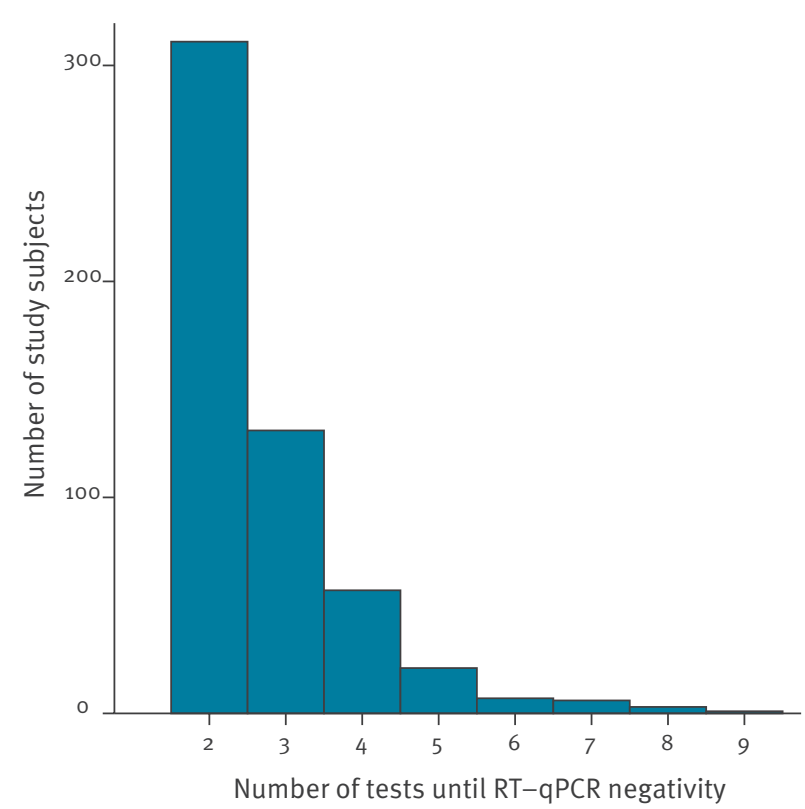

B.

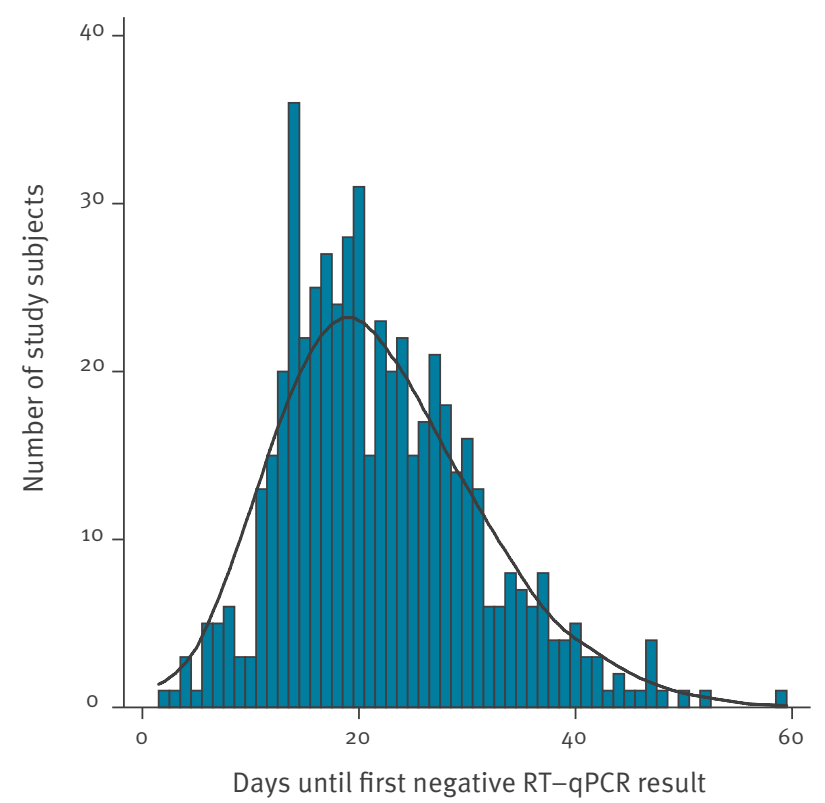

A. Number of samples for RT-qPCR from respiratory material taken until first negative result. More than half of the patients were already negative for SARS-CoV-2-RNA at the time of the second test (percentile $(P)=2$ tests) and $75 \%$ at the third PCR-test (interquartile range: $2-3$ tests). The remaining quarter of the study population needed up to nine tests to become RT- $q P C R-n e g a t i v e ~\left(P_{0.85}=4 ; P_{0.95}=5 ; P_{0.99}=7\right.$; maximum $=9$ tests).

B. Days from onset of symptoms until first SARS-CoV-2 RT-qPCR-negative swab from respiratory material. Grey line: kernel density plot (Epanechnikov). Half of the population tested negative on the 2oth day (interquartile range: day 16-28) after onset of symptoms; the earliest negative test was on the second and the latest negative test on the 68th day after onset of symptoms; one subject with first negative test result beyond 60 days was excluded for more condensed display of distribution.

home isolation currently recommended in Germany [18], $53.5 \%$ of COVID-19 patients still had detectable SARS-CoV-2 RNA (Figure 2). Repeating the analysis after exclusion of 17 patients with 'equivocal' RT-qPCR results recoded as 'positive' changed our results only marginally: we found between 0.33 and $1.00 \%$ shorter time to SARS-CoV-2 negativity estimates than those displayed in Figure $2 \mathrm{~B}$. Comparing the results of the interval-censored analysis (Figure 2 ) with the distribution of time periods until the first negative test result (Figure 1B) shows that interval censoring gave a ca 5 days shorter estimate for the median RNA detection time ( 14.96 versus 20 days).

In a model adjusting for all exposure characteristics at once, patients in the oldest age group had $14 \%$ shorter median time to PCR negativity than those in the youngest age group (time ratio: $0.86, p=0.037$ ), while no such differences were found for the remaining age groups. Similarly, nursing home residents had $28 \%$ shorter median time to RNA negativity than patients with no specific epidemiological context at time of diagnosis (time ratio: $0.72 ; p=0.013$ ). Hospitalisation before home isolation, by contrast, was associated with $26 \%$ longer duration of RNA positivity compared with patients in home isolation throughout COVID-19 (time ratio: $1.26 ; p=0.049$ ) (Supplementary Table S2). Using a model adjusting for all exposure characteristics at once to predict time to RNA negativity for given population proportions only marginally changed the results from the unadjusted model (Figure 2B, Supplementary Table S3).

\section{Contextualisation of findings}

While several countries recommend negative laboratory testing for particular patient groups before discontinuing isolation [2], there is increasing evidence that the period when viral nucleic acid can be detected may exceed the period of infectivity by far. Studying 77 infector-infectee transmission pairs, He at al. found convincing evidence for substantial pre-symptomatic transmission that peaks shortly before the onset of symptoms and then declines until Day 8 after symptom onset [3]. Support for an end of transmissibility around Day 8 after onset of symptoms comes from two independent studies that were not able to propagate virus in cell cultures from RT-qPCR-positive individuals beyond that point in time $[19,20]$. Against this background, and irrespective of the potential reason for this discrepancy (i.e. higher sensitivity of RT-qPCR or detection of non-replicative RNA remnants), our finding that $78 \%$ of patients in home isolation remained RT-qPCR-positive beyond Day 8 after onset of symptoms strongly supports that in patients with mild COVID-19, positive nucleic acid testing results do not allow any conclusions on infectivity. Similarly, 
Duration of SARS-CoV-2-RNA positivity in COVID-19 patients in home isolation, Rhineland-Palatinate, Germany, 2020 $(\mathrm{n}=537)$

A.

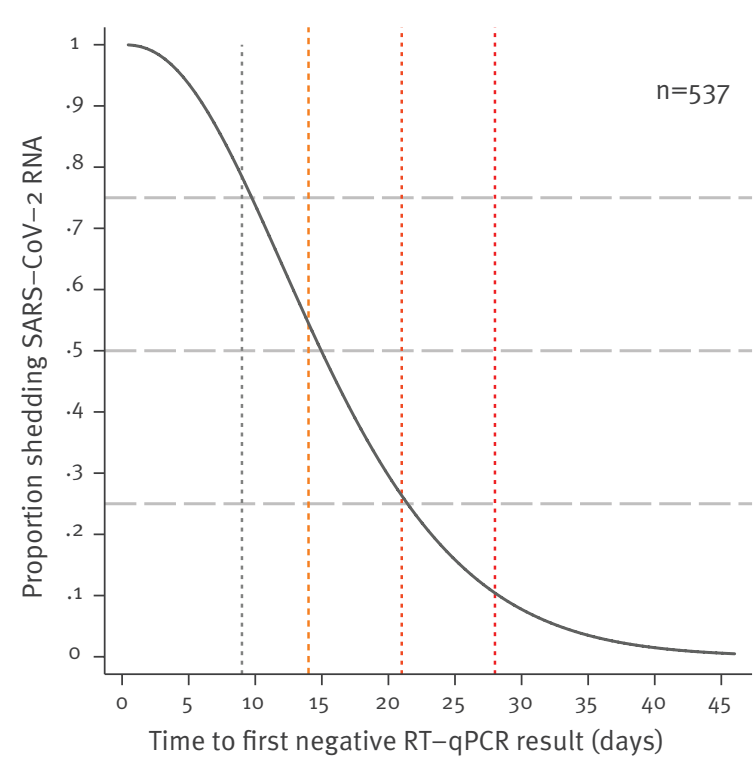

B.

\begin{tabular}{|l|c|c|}
\hline Proportion & Days & $95 \% \mathrm{Cl}$ \\
\hline .99 & 2.24 & $1.88-2.61$ \\
\hline .975 & 3.31 & $2.85-3.77$ \\
\hline .95 & 4.48 & $3.89-5.07$ \\
\hline .90 & 6.15 & $5.33-6.97$ \\
\hline .75 & 9.74 & $8.42-11.07$ \\
\hline .50 & 14.96 & $13.01-16.90$ \\
\hline .25 & 21.43 & $18.96-23.91$ \\
\hline .10 & 28.28 & $25.50-31.06$ \\
\hline .05 & 32.82 & $29.93-35.72$ \\
\hline .025 & 37.01 & $34.03-40.00$ \\
\hline .01 & 42.16 & $39.04-45.29$ \\
\hline
\end{tabular}

$\mathrm{Cl}$ : confidence interval; SARS-CoV-2: severe acute respiratory syndrome coronavirus-2.

Cumulative proportion of SARS-CoV-2 RNA detection in upper respiratory tract samples at various time points after onset of symptoms; estimates from interval-censored survival analysis based on data from 537 symptomatic COVID-19 patients and univariable generalised gamma accelerated failure time regression model.

A. Cumulative proportion of population detection SARS-CoV-2 RNA as a function of time onset of symptoms. Horizontal dashed reference lines at 75, 50 and 25 population percentiles; vertical dotted reference lines at 9 days (grey), 14 days (yellow, i.e. minimum length of home isolation in Germany by 13 June 2020 [18]), 21 days (orange) and 28 days (red). Current best estimate of duration of infectiousness: 8 days $[3,19,20]$.

B. Estimates of 'days after symptom onset' with $95 \% \mathrm{CI}$ for detection of SARS-CoV-2 RNA in cumulative population proportions; Proportion = proportion of population positive for SARS-CoV RNA.

respectively $50 \%, 25 \%$ and $10 \%$ of all patients would be kept overly long at home when choosing the end of the infectious period on Days 14, 21 and $28(+/-2$ days) after onset of symptoms and hereby cautiously taking into account evidence from one report on prolonged detection of replicative virus in one mild case of COVID-19 [21]. Therefore, RT-qPCR-guided containment leads to additional costs through prolonged periods of isolation and repeated sampling and increases the individual and social burden of the current epidemic.

Compared with published estimates on duration of RNA detection after onset of symptoms, ranging from 14 to 24 days for the median and 15.67 to 24.5 days for the mean, our estimate of the mean of 14.96 days is at the lower end. It is difficult to tell whether this is a result of differences in study population or design, or both. From a methodological point of view, however, we expected that our analysis using intervalcensoring (i.e. the interval between the last positive and the first negative swab) would reduce systematic information bias compared with estimates derived from time to first negative swab and would thus lead to shorter estimates of time to RNA negativity than most of the reviewed studies. Accordingly, we found that in our population and setting, the median duration of RNA detection using interval-censoring was ca 5 days shorter than the median time to the first negative test (14.96 versus 20 days).

\section{Conclusion}

RT-qPCR testing seems to be of limited value in guiding the duration of home isolation in mild COVID-19. Negative follow-up testing may be useful in providing certainty about non-infectiousness before ending the isolation in settings where onward transmission has particularly detrimental consequences (e.g. healthcare setting) or in patient groups with known risk factors for prolonged viral shedding and the associated risk of infectiousness (e.g. the severely immunosuppressed). 
For cases where laboratory monitoring is indispensable, knowledge of the RT-qPCR threshold cycle may improve our judgement on whether a positive result indicates infectiousness or not [20]. By contrast, positive follow-up tests correlate poorly with infectiousness. Hence, after the diagnosis of COVID-19 is established, the large proportion of positive results that emerge from RT-qPCR monitoring during the first 4 weeks after onset of symptoms, and that exceed the period of infectiousness by far, do not meaningfully contribute to the anti-epidemic management of mild COVID-19 cases. Instead, they increase the overall cost and social burden of the epidemic without mitigating it. We conclude that for most patients with mild COVID19 , the use of fixed time periods, based on sound estimates of the infectious period, seem more appropriate in guiding the duration of containment measures than laboratory-based approaches. More research on risk groups and factors associated with prolonged infectiousness (e.g. immunosuppression, age and disease severity) is needed to tailor fixed time periods to various settings and groups and ultimately further reduce the use of RT-qPCR in guiding the duration of SARSCoV-2 containment.

\section{Acknowledgments}

Further collaborators of the Palatina Public Health study group at District Public Health Authorities (DPHA) were Lina Boeck, DPHA, 56410 Montabaur; Annette Benzmüller, Svenja Dockendorf, Claudia Leineweber, Christina Klein, Harald Michels, Christian Spies, DPHA, 54292 Trier; Anette Georgens, Anja Hauser, Harald Kreulich, Heiko Magin, Frank Wenner, DPHA, 76726 Germersheim; Michael Heumann, DPHA, 56564 Neuwied; Samira Meiers, DPHA, 56130 Bad Ems; Christine Knauer, Ingeborg Hübner-Zimmermann, Sabine Haag, DPHA, 67059 Ludwigshafen am Rhein; Christiane Steinebrei, DPHA, 67655 Kaiserslautern; Andrea Missal, Danilo Bäcker, Claudia Göttel, DPHA, 66869 Kusel; ErnstDieter Lichtenberg, Silke Hey, DPHA, 55543 Bad Kreuznach; Dietmar Hoffmann, DPHA, 55116 Mainz; Ralf Krauspe, DPHA, 56812 Cochem-Zell; Susanne Kämmerer, Herbert Kreher, DPHA, 66953 Pirmasens.

Funding: No particular funding was received for the conduct of this work.

\section{Conflict of interest}

None declared.

\section{Authors' contributions}

PZ designed and coordinated the study, did the statistical analysis, wrote a first draft and coordinated the finalisation of the manuscript, with support of SC and TB.

SO identified, reviewed, and entered data of COVID-19 patients from records, interpreted the results of the analysis and contributed to the first draft and the final version of the manuscript.

CB, SBe, SBa, SP, CT, HH, HCS, MF, UK, CJ, EH, identified, reviewed, and entered data of COVID-19 patients from records, helped in interpreting the results of the analysis, and contributed to the final version of the manuscript.
$\mathrm{MV}$ and $\mathrm{KJ}$ interpreted the results of the analysis and contributed to the final version of the manuscript.

Collaborators of the Palatina Public Health study group identified, reviewed, and entered data of COVID-19 patients from records at district public health authorities.

\section{References}

1. Wu Z, McGoogan JM. Characteristics of and important lessons from the coronavirus disease 2019 (COVID-19) outbreak in China: summary of a report of 72314 cases from the Chinese Center for Disease Control and Prevention. JAMA. 2020;323(13):1239. https://doi.org/10.1001/jama.2020.2648 PMID: 32091533

2. European Centre for Disease Prevention and Control (ECDC). Guidance for discharge and ending isolation in the context of widespread community transmission of COVID-19. Stockholm: ECDC; 8 Apr 2020. Available from: https://www.ecdc.europa.eu/en/publications-data/ covid-19-guidance-discharge-and-ending-isolation

3. He X, Lau EHY, Wu P, Deng X, Wang J, Hao X, et al. Temporal dynamics in viral shedding and transmissibility of COVID-19. Nat Med. 2020;26(5):672-5. https://doi.org/10.1038/s41591020-0869-5 PMID: 32296168

4. Li N, Wang X, Lv T. Prolonged SARS-CoV-2 RNA shedding: Not a rare phenomenon. J Med Virol. 2020;jmv.25952. https://doi. org/10.1002/jmv.25952 PMID: 32347980

5. Zhou F, Yu T, Du R, Fan G, Liu Y, Liu Z, et al. Clinical course and risk factors for mortality of adult inpatients with COVID-19 in Wuhan, China: a retrospective cohort study. Lancet. 2020;395(10229):1054-62. https://doi.org/10.1016/S01406736(20)30566-3 PMID: 32171076

6. Xiao AT, Tong YX, Zhang S. False negative of RT-PCR and prolonged nucleic acid conversion in COVID-19: Rather than recurrence. J Med Virol. 2020;jmv.25855. https://doi. org/10.1002/jmv.25855 PMID: 32270882

7. Xiao AT, Tong YX, Zhang S. Profile of RT-PCR for SARS-CoV-2: a preliminary study from 56 COVID-19 patients. Clin Infect Dis 2020; ciaa460. https://doi.org/10.1093/cid/ciaa460 PMID: 32306036

8. Yan D, Liu XY, Zhu YN, Huang L, Dan BT, Zhang GJ, et al. Factors associated with prolonged viral shedding and impact of lopinavir/ritonavir treatment in hospitalised non-critically ill patients with SARS-CoV-2 infection. Eur Respir J. 2020;56(1):2000799. https://doi. org/10.1183/13993003.00799-2020 PMID: 32430428

9. Fang Z, Zhang Y, Hang C, Ai J, Li S, Zhang W. Comparisons of viral shedding time of SARS-CoV-2 of different samples in ICU and non-ICU patients. J Infect. 2020;81(1):147-78. https://doi. org/10.1016/j.jinf.2020.03.013 PMID: 32209381

10. Xu K, Chen Y, Yuan J, Yi P, Ding C, Wu W, et al. Factors associated with prolonged viral RNA shedding in patients with COVID-19. Clin Infect Dis. 2020;ciaa351. https://doi. org/10.1093/cid/ciaa351 PMID: 32271376

11. Hu X, Xing Y, Jia J, Ni W, Liang J, Zhao D, et al. Factors associated with negative conversion of viral RNA in patients hospitalized with COVID-19. Sci Total Environ. 2020;728:138812. https://doi.org/10.1016/j. scitotenv.2020.138812 PMID: 32335406

12. Qi L, Yang Y, Jiang D, Tu C, Wan L, Chen X, et al. Factors associated with the duration of viral shedding in adults with COVID-19 outside of Wuhan, China: a retrospective cohort study. Int J Infect Dis. 2020;96:531-7. https://doi.org/10.1016/j. ijid.2020.05.045 PMID: 32425636

13. Noh JY, Yoon JG, Seong H, Choi WS, Sohn JW, Cheong HJ, et al. Asymptomatic infection and atypical manifestations of COVID-19: Comparison of viral shedding duration. J Infect. 2020;S0163-4453(20)30310-8. https://doi.org/10.1016/j. jinf.2020.05.035 PMID: 32445728

14. Zhang Z, Sun J. Interval censoring. Stat Methods Med Res. 2010;19(1):53-70. https://doi.org/10.1177/0962280209105023 PMID: 19654168

15. Lindsey JC, Ryan LM. Tutorial in biostatistics methods for interval-censored data. Stat Med. 1998;17(2):219-38. https:// doi.org/10.1002/(SICI)1097-0258(19980130)17:2く219::AIDSIM735’3.0.CO;2-O PMID: 9483730

16. Akaike $H$. A new look at the statitstical model identification. IEEE Trans Automat Contr. 1974;19(6):716-23. https://doi. org/10.1109/TAC.1974.1100705

17. Turnbull BW. The empirical distribution funtion with arbitraily grouped censored and truncated data. J R Stat Soc B. 
1976;38(3):290-5. https://doi.org/10.1111/j.2517-6161.1976. tb01597.x

18. Robert Koch-Institut (RKI). COVID-19: Kriterien zur Entlassung aus dem Krankenhaus bzw. aus der häuslichen Isolierung.

[Criteria for discharge from hospital or home-isolation]. Berlin: RKI. [Accessed: 13 Jun 2020].

19. Wölfel R, Corman VM, Guggemos W, Seilmaier M, Zange S, Müller MA, et al. Virological assessment of hospitalized patients with COVID-2019. Nature. 2020;581(7809):465-9. https://doi.org/10.1038/S41586-020-2196-x PMID: 32235945

20. Bullard J, Dust K, Funk D, Strong JE, Alexander D, Garnett L, et al. Predicting infectious SARS-CoV-2 from diagnostic samples. Clin Infect Dis. 2020;ciaa638. https://doi.org/10.1093/cid/ ciaa638 PMID: 32442256

21. Liu WD, Chang SY, Wang JT, Tsai MJ, Hung CC, Hsu CL, et al. Prolonged virus shedding even after seroconversion in a patient with COVID-19. J Infect. 2020;81(2):318-56. https://doi. org/10.1016/j.jinf.2020.03.063 PMID: 32283147

\section{License, supplementary material and copyright}

This is an open-access article distributed under the terms of the Creative Commons Attribution (CC BY 4.0) Licence. You may share and adapt the material, but must give appropriate credit to the source, provide a link to the licence and indicate if changes were made.

Any supplementary material referenced in the article can be found in the online version.

This article is copyright of the authors or their affiliated institutions, 2020. 